\title{
Traduire
}

Ine autre perspective sur I t raduction

Revue française de la traduction

$226 \mid 2012$

Face au miroir

\section{La traduction comme substitut d'amour, thérapie et art de la relation}

La représentation de la traductrice fictive. Analyse de sept cas

\section{Anja Siouda}

\section{OpenEdition}

\section{Journals}

Édition électronique

URL : http://journals.openedition.org/traduire/138

DOI : $10.4000 /$ traduire.138

ISSN : 2272-9992

\section{Éditeur}

Société française des traducteurs

Édition imprimée

Date de publication : 1 janvier 2012

Pagination : 21-38

ISBN : 039-773X

ISSN : 0395-773X

\section{Référence électronique}

Anja Siouda, «La traduction comme substitut d'amour, thérapie et art de la relation », Traduire [En

ligne], 226 | 2012, mis en ligne le 07 août 2016, consulté le 10 octobre 2020. URL : http:// journals.openedition.org/traduire/138; DOI : https://doi.org/10.4000/traduire.138 


\section{La traduction comme substitut d'amour, thérapie et art de la relation La représentation de la traductrice fictive. Analyse de sept cas (1)}

\section{Anja Siouda}

L'idée de ce mémoire m'est venue après la lecture du roman La traduction est une histoire d'amour du romancier canadien Jacques Poulin. En effet, ne devrions-nous pas tous, traductrices et traducteurs, spécialistes et amoureux du langage par excellence, être touchés voire subjugués par un pareil titre ? Quel métier de rêve exerçons-nous donc ? L'amour nous fait-il gagner notre pain quotidien ?(2) L'image des traducteurs et traductrices, telle qu'elle est majoritairement représentée dans la littérature, correspond-elle à cet idéal à l'eau de rose évoqué par le titre du roman de Poulin ou à une description nettement plus sombre ? Peut-on comparer fiction et réalité ? Y a-t-il des différences de représentation selon qu'il s'agit d'un traducteur ou d'une traductrice?

Le sujet méritait d'être approfondi, mais comme pour toute recherche, il fallait d'abord le délimiter. Je souhaitais contribuer à la traductologie féministe, j'ai donc choisi des protagonistes féminins, soit sept traductrices héroïnes de sept textes très différents. Je me proposais de comparer l'image de la traductrice fictive à celle du traducteur fictif, de dénicher les éventuels clichés sexistes et de voir si les réflexions et comportements des traductrices fictives avaient un rapport avec la biographie des auteur-e-s puisque cinq des sept auteur-e-s sont traducteurs/traductrices eux-/elles-mêmes. II est à noter que les sept textes choisis sont très hétérogènes et les réflexions sur la traduction n'y sont pas abordées dans la même mesure. Comme l'indique déjà le titre La traduction est une histoire d'amour (Poulin) et Traductrice de sentiments (Rioux) sont des romans dont le sujet principal est le processus de traduction et les réflexions s'y rapportant. II en va de même pour A.S. (Wolter), bien que le titre ne l'indique

(1) Cet article est un résumé du mémoire de traductologie, rédigé en allemand et présenté par l'auteure à l'École de traduction et d'interprétation de Genève (ETI) en juin 2010. Le texte intégral du mémoire peut être téléchargé gratuitement à l'adresse : $\underline{\text { http://www.anjasiouda.com/index.php/publikationen. }}$

(2) On pourrait aussi, ironiquement, rappeler à ce propos la comparaison célèbre de Louis Truffaut (22 octobre 1992), encore appréciée et citée par certains traductologues : "La traduction partage avec une autre profession - moins intellectuelle - le privilège d'être l'un des métiers les plus vieux du monde ". 
pas. Dans Transgressions (Dunant) et Juste à la porte du jardin d'Eden (Reznikov), le processus de traduction est également abordé mais occupe une place plus modeste. Dans Transgressions il est même instrumentalisé par l'auteure pour augmenter le suspense. Dans Die Ferienfamilie (Frischmuth) et The Translator (Aboulela) par contre, le processus de traduction et l'activité de la traductrice restent marginaux. En effet, les héroïnes dans ces deux romans peuvent être interprétées comme des Über-Setzerinnen(3) au sens métaphorique du terme.

\section{1. État de la recherche}

\subsection{Un sujet récent}

En juin 2010, lorsque j'ai soutenu le mémoire dont je vous résume l'essentiel ici, le sujet de la représentation du traducteur/de la traductrice dans la littérature était considéré comme assez récent, même si quelques recherches plus approfondies avaient déjà été menées sur ce thème. C'est Klaus Kaindl, avec ses deux ouvrages collectifs Wortklauber, Sinnverdreher, Brückenbauer (2005) et Helfer, Verräter, Gaukler (2008) qui, le premier, s'est intéressé à cette question mais Dörte Andres y a consacré sa thèse d'habilitation, Dolmetscher als literarische Figuren: von Identitätsverlust, Dilettantismus und Verrat (2008) et Christine Wilhelm a également choisi ce sujet pour sa thèse, Traduttore-traditore, Vermittler durch Verrat. Grenzgänger zwischen Vermittlung und Verrat. Eine Betrachtung literarischer Übersetzer- und Dolmetscherfiguren (2010). Un projet de recherche initié en 2009 par Jean Delisle (et maintenant placé sous la direction de Patricia Godbout), porte sur Les traducteurs fictifs dans la littérature québécoise depuis 1960 et se propose de brosser un portrait du traducteur tel qu'il se dégage de cette littérature(4).

En 2005, Klaus Kaindl cherchait plutôt à comparer la pratique du traducteur fictif à celle du traducteur réel mais, par la suite, les chercheurs ont eu tendance à examiner le rôle et l'image attribués au traducteur fictif ainsi que la raison pour laquelle le personnage fictif du traducteur/ de la traductrice est populaire.

En automne 2011, Klaus Kaindl a également mis sur pied la First International Conference on Fictional Translators qui s'est tenue à Vienne.

(3) Du verbe allemand übersetzen qui veut dire à la fois traduire et passer d'une rive à une autre. Ici, le mot traductrice a donc plutôt le sens de médiatrice entre deux cultures (The Translator) ou entre différentes conceptions du monde (Die Ferienfamilie).

(4) Voir les articles de Jean Delisle et de Patricia Godbout dans ce même numéro, respectivement en page 7 et 39. 


\subsection{La traduction comme motif favori et métaphore}

Pourquoi le processus de traduction et la traductrice ou le traducteur sont-ils devenus des motifs littéraires favoris ? De fait, le traducteur est devenu un sujet d'analyse depuis qu'Antoine Berman s'est focalisé sur " la position traductive " et " la pulsion à traduire " en 1995. Jiř́ Levý s'était, d'ailleurs, déjà intéressé bien plus tôt au processus de traduction (dès 1967). Comme la plupart des auteur-e-s qui choisissent des protagonistes traducteurs/traductrices sont eux/ elles-mêmes familiarisés avec la traduction, il n'est guère étonnant qu'ils attribuent un rôle central au traducteur/à la traductrice, comme le fait la traductologie depuis bien des années.

En outre, dans un monde de plus en plus global, il n'est pas surprenant que les expériences humaines par rapport aux langues et aux cultures deviennent un sujet récurrent dans la littérature. Alors que, par le passé, la traduction a souvent été vue comme un processus de perte de sens, on a plutôt tendance à la considérer désormais comme un processus créatif.

\subsection{Fiction et réalité}

Mais peut-on effectivement comparer fiction et réalité ?

Selon Kaindl, le fait d'attribuer certaines caractéristiques au traducteur fictif n'est pas un hasard. Si cela ne veut pas dire qu'on puisse réduire la littérature au rapport entre représentation fictive et expérience personnelle de la réalité(5), c'est une façon d'avoir " des informations sur des valeurs et des attitudes qui sont plus subtiles et moins aisées à acquérir "(6) (Rockwell 1974 cité par Kaindl, 2008b). Delisle exprime cette idée de la façon suivante : "Le sujet traduisant, tout comme l'écrivain, est porteur des représentations symboliques de sa société " (Delisle 2002 : 2) et il dit aussi : " La fiction, au fond, ce n'est pas le mensonge, c'est mentir pour dire vrai „ (Delisle 2003 : 19). Ailleurs, Delisle précise que

[... ] les traducteurs de papier ne manquent pas de nous livrer leurs sentiments sur leur métier. Et il n'est pas défendu de croire que ces sentiments sont partagés par les auteurs eux-mêmes. Pour certains, la traduction est un havre de paix et une oasis de tranquillité [...]. D'autres personnages, en revanche, sont agacés d'être obligés de traduire, soit qu'ils ne se sentent pas à la hauteur de la tâche, soit que cette activité nuise à leurs aspirations d'écrivain. (Delisle $2003: 30)$

Indépendamment du rapport entre réalité et fiction, on peut néanmoins constater aisément que certains rôles, attributs et caractéristiques sont prêtés aux traducteurs et traductrices fictifs de manière extrêmement récurrente.

(5) " [...] Literatur [lässt sich] nicht auf die Beziehung zwischen fiktionaler Darstellung und persönlicher Realitätserfahrung reduzieren [...]" (Kaindl 2008b : 308).

(6) more subtle and less easily obtained information about values and attitudes. Traduction A. Siouda. 

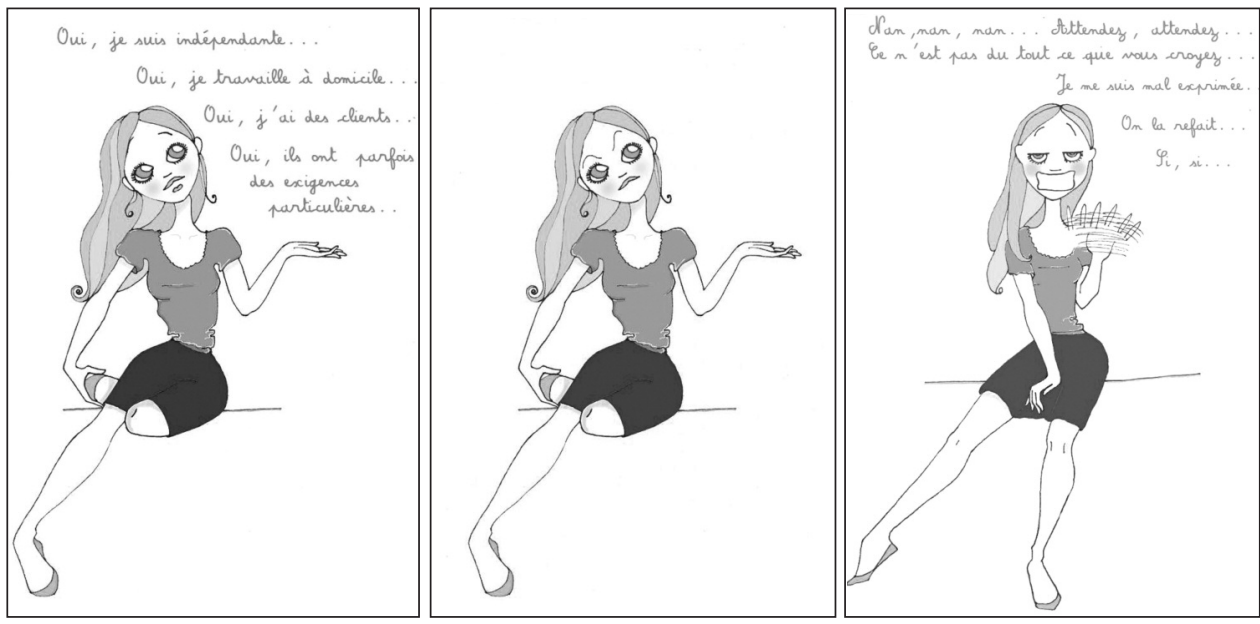

Illustration : Marlène Junius, http://alotoftralala.over-blog.com

\subsection{L'image des traducteurs/interprètes selon KaindI}

Quelle est donc l'image la plus répandue des traducteurs et traductrices fictifs ?

Kaindl donne une longue liste de thèmes, d'idées et de clichés où l'on trouve entre autres: déracinement, isolation, marginalité, aliénation, perte de la langue maternelle, dépendance, pouvoir et abus de pouvoir, mais aussi fidélité inconditionnelle envers l'auteur et le texte original, perte d'identité, " bonne à tout faire ", perroquet, machine à traduire, etc. (voir Kaindl 2005 p. 12 et suivantes). Ce qui frappe ici, c'est que cette longue liste comporte des items majoritairement négatifs. II convient de rappeler qu'elle a été établie après l'analyse de 26 œuvres littéraires diverses, dont seize avaient été éditées entre 1990 et 2005.

Sur la base des exemples issus de la littérature, Kaindl constate aussi que les traducteurs/traductrices apparaissent souvent avec des défauts physiques, des maladies, des handicaps, des faiblesses, mais qu'ils et elles présentent aussi des dysfonctionnements psychiques. En résumé, Kaindl relève " [...] que la traduction est soit une maladie résultant d'une approche pathologique de la réalité et de conflits d'identité. Ou alors que la traduction rend malade "(7).

Ailleurs, Kaindl précise que l'image et le statut d'un banal prestataire de service qu'on attribue au traducteur/à la traductrice contraste avec le niveau de formation supérieur de bon nombre de ces professionels qui sont bardés de diplômes universitaires. La différence entre la perception par la société et l'estime de soi du traducteur/de la traductrice conduit à la frustration et à l'insatisfaction.

(7) "[...] dass Translation entweder eine Krankheit ist, die aus pathologischen Realitätszugängen und Identitätskonflikten resultiert. Oder aber, dass Translation krank macht ". (Kaindl 2008 : 315). Traduction A. Siouda. 


\section{Analyse de sept cas}

\subsection{Brève description des textes analysés}

Jacques Poulin *1937 (traducteur, écrivain) La traduction est une histoire d'amour (TA) Marine, la très jeune narratrice, est invitée par Waterman, l'auteur du roman La piste de l'Oregon, à traduire son œuvre de l'anglais vers le français. II l'invite à vivre dans son chalet sur l'Île d'Orléans, tandis que lui-même vit dans une tour au Québec. Une relation amicale s'établit entre eux et ils sauvent une adolescente suicidaire qu'ils " adoptent ".

\section{Hélène Rioux *1949 (traductrice, écrivaine) Traductrice de sentiments (TS)}

Éléonore, la traductrice, est une femme esseulée et déprimée âgée de 35 ans qui est dégoûtée de traduire des romans à l'eau de rose. Elle se sent coupable de transmettre l'image fausse des relations d'amour de Don Juan galants et se rend en Espagne pour traduire l'autobiographie de Leonard Ming, un serial killer sadique. Elle espère rencontrer la mort et sa vérité à travers le texte. Sa propre fille qu'elle a donnée en adoption est morte à l'âge de 3 ans.

\section{Christine Wolter *1939 (traductrice, écrivaine) A.S. (AS)}

La narratrice Möbius est traductrice de textes littéraires. Dans une lettre à un ami, elle décrit ses réflexions par rapport au processus de traduction qu'elle est en train de vivre avec une œuvre d'A.S. (le lecteur devine qu'il s'agit du célèbre auteur Alberto Savinio). Elle vit avec son fils Robert et son compagnon Roberto qui n'est pas le père de l'enfant. Le processus de traduction est vécu de façon très contradictoire, conflictuelle et émotionnelle.

\section{Sarah Dunant *1950 (journaliste, écrivaine) Transgressions (TG)}

La traductrice Elizabeth Skvorecky vit seule dans une maison à Londres, en compagnie de son chat. Elle traduit de la littérature trash (pornographique) du tchèque vers l'anglais. Dans sa vie réelle, elle se fait violer et ressent une certaine volupté avec son violeur, qui est inexpérimenté. Pour pouvoir le dénoncer à la police, elle utilise ses traductions (modifiées pour l'appâter) qu'elle met dans sa poubelle pour que le violeur les trouve. II revient effectivement et elle finit par le tuer in extremis.

Patricia Reznikov *1962 (illustratrice, écrivaine) Juste à la porte du jardin d'Eden (JPE) Colomba est une traductrice qui aspire à devenir écrivaine. Elle traduit des romans policiers et passe sa vie avec un groupe d'amis trentenaires. Nadja, une amie mystérieuse, s'introduit dans le groupe et détruit l'équilibre des amitiés. Colomba y reste insensible car elle a commencé à correspondre avec Paul Ashburn, romancier et poète américain qu'elle adore et dont elle aimerait traduire l'unique roman. Le projet de traduction lui échappe et la rencontre avec l'écrivain échoue, car il meurt avant l'arrivée de Colomba aux États-Unis. 


\section{Barbara Frischmuth *1941 (traductrice, écrivaine) Die Ferienfamilie (FF)}

Nora est traductrice et mère d'un fils de six ans, Pu. Elle loue une maison pour y passer l'été avec son fils, le fils de son premier mari, Fenek, et Laja, la fille de sa sœur. Elle passe des vacances tout en travaillant. Grâce à son talent de communicatrice, elle maîtrise le groupe d'enfants et leurs relations familiales perturbées. Une amitié se noue entre Nora et un ancien camarade de classe, Lajosch. Les vacances se terminent abruptement : Fenek a un accident, ce qui fait venir sa mère d'Australie.

\section{Leila Aboulela *1964 (statisticienne, écrivaine) The Translator (TT)}

Sammar est une jeune traductrice musulmane déprimée qui traduit des textes de terroristes de l'arabe vers l'anglais. Elle souffre de la perte de son mari Tarig, mort dans un accident de voiture, et de l'absence de son fils qu'elle fait garder par sa belle-mère à Khartoum. Elle tombe amoureuse de l'islamologue Rae et espère sa conversion à l'islam pour pouvoir se marier avec lui. Comme Rae ne se convertit pas, elle retourne à Khartoum. Finalement, elle apprend la conversion de Rae par courrier, et un avenir commun se dessine.

\subsection{La traductrice et sa relation avec l'auteur}

II est à noter que le cas d'une relation d'un traducteur avec l'auteure d'un texte ne figure ni parmi les textes étudiés dans le présent travail, ni dans les diverses recherches s'y rapportant.

Dans le présent corpus, la traductrice, par contre, noue très souvent une relation directe ou indirecte avec le texte à traduire et/ou avec son auteur (qui le représente).

Cette relation constitue même le sujet principal dans les textes de Poulin, Rioux et Wolter. Tout tourne autour de l'auteur du texte de départ et de ce texte, et il n'y a pas de véritable action ou intrigue.

\subsubsection{L'auteur comme idole et son texte de départ comme expérience érotique sublimée}

L'idolâtrie de la traductrice face à l'auteur, voire même la fusion (platonique) entre traductrice et auteur est particulièrement évidente dans le texte de Jacques Poulin, La traduction est une histoire d'amour. Traductrice et auteur sont déjà liés, ne serait-ce que par leur noms : Marine et Waterman. L'élément eau les lie donc dès le départ tout comme leur amour mutuel des chats. Au fil de l'histoire, il s'établit une sorte de relation père-fille (reflétant aussi la hiérarchie, la disparité entre auteur et traductrice), Waterman étant le protecteur de Marine : il la tutoie, elle le vouvoie. Lori Saint-Martin s'interroge ainsi sur cette relation :

Quelle est donc la nature du lien entre l'écrivain déjà âgé et sa jeune traductrice? Globalement positive, elle comporte néanmoins des éléments troublants dont le moindre n'est pas justement leur relation professionnelle : en effet, le rapport auteur/traducteur peut rappeler le rapport hiérarchique entre masculin et féminin. [... . Marine est seconde par rapport à celui dont l'œuvre l'inspire. (Saint-Martin 2007 : 42) 
Le fait que Marine se trouve en deuxième position, comme la traduction face à l'original, reflète le cliché tenace de la traduction comme simple copie et, de surcroît, l'image de la femme dépendante de l'homme. Cette idée rappelle l'idée de la création biblique (et coranique) où Ève est créée à partir d'une côte d'Adam.

Borek le formule ainsi :

La traduction qui disparaît derrière l'original, le traducteur qui est en retrait par rapport à l'auteur, c'est le renvoi décrété de la copie femme au second plan de l'original homme. C'est l'interprétation de la genèse selon Thomas d'Aquin se référant à Aristote. (8)

Cette idée de la traduction comme copie contraste fortement avec la conception moderne de la traduction, considérée comme une nouvelle création ou comme une production intertextuelle, au même titre que l'original.

Dans ce roman, l'auteur Poulin ne fait que perpétuer des clichés sexistes sur les femmes : les femmes sont des êtres nus, naifs, enfantins, obéissant à leur instinct. Elles sont à la recherche d'un protecteur qu'elles adulent, ne s'intéressent pas au sexe, ont forcément subi des abus sexuels dans leur enfance, se culpabilisent. Ainsi, Marine est infantilisée, car elle ne parle qu'à Waterman, aux petites filles, aux animaux, aux plantes et à elle-même. Elle n'a aucun contact avec des personnes de son âge et souffre d'une mauvaise image d'elle-même, d'un manque de confiance. Elle ne veut se lier avec personne, se sent comme une étrangère sur terre et se compare à Isabelle Eberhardt. Elle se met par contre dans la peau de Waterman en enfilant ses habits à lui. Si Waterman est présenté comme un père bienveillant, le roman ne manque pas d'allusions érotiques, dès la première phrase (c'est Marine, la narratrice qui " parle ") :

Nue comme une truite, je sortis de l'étang avec une poignée d'algues dans chaque main, lorsque tout à coup, je vis ma chatte se ruer tête baissée vers une petite chose noire qui descendait la côte menant au chalet. (TA p. 11)

Poulin aurait pu écrire chat au lieu de chatte, le sexe de l'animal ne jouant aucun rôle dans la suite de l'histoire. Plus loin, on trouve une autre allusion : " Pour me dire qu'il avait assisté à la poursuite, il mima avec ses hanches le mouvement ondulatoire de la chatte obèse [...] " (TA p. 11). À un moment dans l'histoire, Marine a un accident et c'est Waterman qui la soulève et la porte jusqu'à la maison, la met au lit, la frictionne. II n'y a certes pas de rapport sexuel, mais l'érotisme sous-jacent est bien là et les deux protagonistes le subliment ainsi :

Selon une croyance répandue, la meilleure manière d'apprendre une langue c'est d'avoir une relation amoureuse avec un locuteur natif de cette langue. L'acte de traduire va plus loin que cela, permettant au traducteur d'avoir une relation amoureuse avec les mots de l'auteur. II y a un certain lien entre passion et langue, entre expérience érotique et manipulation physique des

(8) Das Verschwinden der Übersetzung hinter dem Original, das Zurücktreten des Übersetzers hinter dem Autor ist das verordnete Zurücktreten der Zweitschrift Frau hinter die Originalausgabe Mann. Dies ist die auf Aristoteles sich berufende Lesart der Genesis von Thomas von Aquin. (Borek 1996 : 32). Traduction A. Siouda. 
mots. Les mots font appel au sens et ont une qualité voluptueuse [... ] dans la traduction, tout tourne autour de la séduction et de l'attirance : la traductrice se sent attirée par la langue et par le texte. Amoureuse des mots et des nouveaux mots complexes qu'ils créent, elle est séduite par la langue étrangère et cela lui donne du plaisir. (9)

On pourrait en conclure que cette "love affair with the author's words", cette "connection between passion and language, between erotic experience and the physical manipulation of words " se retrouve chez le couple Waterman/Marine et qu'elle constitue le substitut d'une relation sexuelle réelle.

\subsubsection{L'auteur comme monstre et son texte de départ comme thérapie}

Au niveau du nom des protagonistes, on peut également constater une certaine fusion dans le roman rédigé par Hélène Rioux, Traductrice de sentiments. Éléonore la traductrice et Léonard, le sérial killer et auteur du texte autobiographique de départ, ont des noms apparentés.

La parenté des deux prénoms laisse entendre qu'entre elle et lui s'est établie une complicité qui est la raison même du récit. Éléonore vit les passions cruelles du meurtrier comme des fantasmes personnels, meurtrière et victime elle-même, comme si elle avait à exorciser une très ancienne tragédie dont les actes essentiels lui échappent.(10)

La traductrice qui n'a traduit que des romans à l'eau de rose (" Je vivais dans les limbes, traduisant comme une machine d'insipides romans. " - TS p. 24) finit par s'enfoncer dans la culpabilité et la dépression. C'est dans cet état qu'elle s'envole pour l'Espagne pour y traduire l'autobiographie de Léonard Ming et finit par s'identifier à la bestialité de l'auteur.

Le roman Traductrice de sentiments dans son entier est imprégné de morbidité (chaque chapitre est précédé de citations des cahiers de Nijinski), la traductrice Éléonore se sent " aussi nue que la mer. Aussi seule. " (TS p. 31) et elle se voit quasiment morte : "J'aurais aussi bien pu me laisser mourir d'inanition. Ma vie se limitait à son ersatz, telle qu'une ligne qui vibre à peine sur un écran à l'hôpital, dans la salle des soins intensifs. J'étais sans courage. " (TS p. 33). Malgré cela, la traductrice paraît parfois assez sûre d'elle quand il s'agit de son travail. Ainsi désigne-t-elle son activité non comme traduction, mais comme écriture. Dans l'avion qui l'emmène en Espagne, elle tombe même sur une de "ses lectrices " (TS p. 19). Rioux fait donc disparaître la démarcation traditionnelle entre écriture et traduction, car la traduction aussi est un acte concret, créatif et positif (voir Nutting 2005 : 260).

(9) It is a common belief that the best way to learn a language is by having a love affair with a native speaker of that language. The act of translation goes beyond this, allowing the translator to have a love affair with the author's words. There is a certain connection between passion and language, between erotic experience and the physical manipulation of words. Words appeal to the senses and have a voluptuous quality [...] translation is all about seduction and attraction: the translator finds herself drawn to the language and attracted to the text. In love with words and the intricate new words they create, she is seduced by the foreign language and this brings her pleasure. (Milano Appel 2003 : 29). Traduction A. Siouda.

(10) Commentaire XYZ éditeur http://www.xyzedit.qc.ca/communique.php?lang=fr\&id=169 page consultée le 23.08.2009 
Contrairement à la fusion entre Marine et Waterman chez Poulin, la fusion entre traductrice et auteur est ici non voulue et terrifiante. Non seulement la traductrice semble perdre sa propre identité, mais elle adopte aussi celle du tueur et de sa victime. Au fil des atrocités relatées par l'auteur, Éléonore finit par souhaiter revenir à sa situation précédente d'automate insensible. Elle pense à renoncer mais finit par terminer la traduction qui devient un best-seller. Éléonore se sent alors coupable de contribuer à la diffusion de cette terrible autobiographie.

Rioux donne une image assez réaliste de la pratique de la traduction. Le conflit d'identité vécu par Éléonore reflète des situations vécues par des traductrices et traducteurs réels confrontés à des textes douteux. Toutefois la description de la traductrice esseulée, angoissée et dépressive est problématique. Éléonore se force à traduire cette autobiographie sadique parce qu'elle voit cette activité comme une sorte de thérapie (qui n'aboutit pas, d'ailleurs, car ses problèmes psychiques s'en trouvent renforcés). Dans la réalité aussi, certains voient la traduction comme une thérapie : "En effet, parfois le fait de traduire un livre peut avoir un effet cathartique provoquant certaines émotions et sentiments qui, tout en étant douloureux, peuvent être thérapeutiques et libérateurs; un peu comme une séance avec un psychanalyste "(11). Éléonore, quant à elle, semble avoir absorbé cette autobiographie comme une éponge, au point où ses propres pensées se confondent avec celles de son auteur : "Sa voix me trouble. A-t-il vraiment écrit ses mots, les ai-je vraiment traduits, ou suis-je en train de les inventer ? "(TS p. 112). Même lorsque la traduction est terminée, ses pensées morbides et résignées ne la lâchent pas.

\subsubsection{Entre dégoût et fascination}

\subsubsection{Aversion, fusion, libération}

Dans A.S. de Christine Wolter, un texte de seulement trente pages, la narratrice et traductrice Möbius passe d'un état de rage et d'aversion à la fusion avec l'auteur, puis finalement à la libération. Lors de la fusion et de l'identification avec l'auteur, elle s'isole totalement de sa famille. Ceci rappelle les conditions réelles des traducteurs et traductrices : "Capacité de traduire en apnée pendant 20 heures d'affilée, 7 jours par semaine, 365 jours par année " (Brisson 2008 : 14).

Ce texte se différencie des autres, car il ne tourne qu'autour de la réflexion de l'héroïne sur le processus de traduction. II n'y a quasiment pas d'action. Le mystérieux A.S., auteur du texte d'origine de la traductrice fictive, est vite démasqué par le lecteur. II s'agit d'Alberto Savinio et de sa Nuova Enciclopedia, ouvrage que Christine Wolter a effectivement traduit en allemand et qui est paru en 1983 sous le titre Neue Enzyklopädie. Évidemment, on peut seulement en conclure que les réflexions de l'héroïne reflètent les pensées et l'état psychique de la traductrice Wolter pendant le processus de traduction. À travers ce texte et son héroïne Möbius, l'au-

(11) "Indeed, at times, translating a book can have a cathartic effect, provoking certain emotions and feelings that, while painful, may be therapeutic and liberating; a little like a session with an analyst ". (Milano Appel 2003 : 30). Traduction A. Siouda. 
teure Wolter s'est créé une soupape efficace pour exprimer son indignation vis-à-vis d'un texte source à caractère parfois ouvertement misogyne qu'elle était obligée de traduire fidèlement.

Möbius n'est pas très sûre d'elle non plus, mais elle n'est ni dépressive ni esseulée, bien au contraire. C'est une femme bien dans sa peau menant de front maternité, relation amoureuse et travail. Réfléchissant au processus de traduction dès le premier paragraphe du texte, elle analyse et résume sa relation avec l'auteur A.S., décédé depuis des années, mais ressuscité à travers le texte de départ et sa traduction.

Sa langue par-dessus ma langue - ceci est peut-être la formule la plus pertinente de ma relation avec A.S. [...] Aujourd'hui que son livre terminé est posé devant moi, je la ressens plus qu'à l'époque, cette intimité de nos deux langues proches. Oui, plus encore, notre nudité mutuelle. En effet, ma relation avec A.S., que j'avais considérée comme un rapport de travail à court terme, s'est transformée au cours d'un surmenage qui ne voulait pas finir en quelque chose d'autre, difficile à décrire, quelque chose qui nous mettait à nu, nous dépouillait, qui nous soumettait l'un à l'autre, qui déchirait, qui ramollissait et qui laissa des traces, lourdes et indélébiles. Pétri l'un par l'autre, on s'est séparé.(12)

Il est frappant que l'on retrouve ici l'idée de nudité. Cette fois, cependant, elle ne concerne pas uniquement la traductrice (comme Marine chez Poulin), mais la traductrice ET l'auteur. Les deux se trouvent nus et dévoilés à la fin du processus de traduction, dans un sens métaphorique et non érotique.

Le jeu de mots Seine Zunge über meine Zunge, (littéralement : sa langue par-dessus ma langue) se réfère évidemment au mot italien lingua qui désigne la langue comme organe et la langue comme idiome, comme en français. En allemand, le terme Zunge renvoie à l'organe... et la phrase évoque à la fois le jeu des langues lors d'un baiser et la substitution de la langue de l'auteur à celle de la traductrice. Ainsi, le texte d'arrivée est toujours dominé par le texte de départ mais comme l'auteur et la traductrice s'influencent mutuellement ("Eins durch den anderen geknetet "), on comprend que l'original et la traduction dépendent l'un de l'autre. Sans original, on ne pourrait d'ailleurs pas parler de traduction et sans traduction, on ne pourrait pas parler d'un original. En plus, comme mentionné plus haut, selon la conception moderne de la traduction, cette dernière constitue une nouvelle interprétation du texte d'origine et une re-création.

(12) Seine Zunge über meine Zunge - dies ist vielleicht die treffendste Formel für mein Verhältnis zu A.S. [...] Heute, da sein Buch fertig vor mir liegt, empfinde ich stärker als früher die Innigkeit unserer Zungen-Nähe. Ja mehr, unsere Nacktheit voreinander. Denn meine Beziehung zu A.S., die ich für ein kurzfristiges Arbeitsverhältnis gehalten hatte, wurde im Lauf einer nicht enden wollenden Überanstrengung zu etwas schwer beschreibbarem anderen, entblösste uns, enthüllte uns, unterwarf eins dem andern, riss auf, machte weich und hinterliess Abdrücke, die schwer und nicht zu löschen sind. Eins durch den anderen geknetet, trennten wir uns. (AS p. 124). Le texte en gras est seulement en italique dans l'original. Traduction A. Siouda. 
Au fil du processus de traduction, la traductrice intériorise de plus en plus le texte de départ. Elle y pense même quand elle fait l'amour avec son partenaire. Plus loin, elle se soumet tant au niveau du contenu que de la forme:

Pendant que j'étais assise, me torturant en déroulant et enroulant ses phrases serpentines afin de ne rien perdre du jeu intentionnellement étincelant, voilà qu'il dit futilement et en passant : ... c'est ainsi, inexactes telles qu'elles sont, les femmes ... je me figeai de rage. [...], j'écrivis donc fidèlement et exactement c'est ainsi, inexactes telles qu'elles sont, les femmes, me levai et courus à la cuisine(13).

On trouve en effet, d'autres passages qui suscitent la rage de la traductrice :

Eh bien, continua A.S., sans me regarder, chez les femmes, on reconnaît plus facilement de quel animal elles descendent, car cette origine n'a pas été effacée comme chez l'homme par la volonté, la conscience et une dure lutte pour la vie.

Je lançai l'édition de la Biblioteca Adelphi volume 70 contre la fenêtre. (14)

Malgré cette rage que la traductrice ressent envers A.S. et ses idées, elle finit par absorber les idées sexistes et par les distiller à sa famille et à ses amis. En fait, dans son cercle d'amis, elle se rend compte qu'A.S. est un auteur très apprécié voire vénéré. Il est très célèbre et elle réalise qu'à travers sa traduction, elle bénéficie d'une partie de sa gloire. Après avoir terminé la traduction, Möbius se sent libérée, et la présence quasi physique de l'auteur du texte d'origine (qui regardait parfois par-dessus son épaule) s'est évaporée.

De mon point de vue, le " métatexte " littéraire A.S. est particulièrement réussi car il intègre à la création littéraire des réflexions personnelles ironiques (et nécessairement tues pour des raisons pragmatiques) en décrivant la réalité du processus de traduction sous toutes ses facettes (conditions de travail de la mère de famille, fusion avec la traduction, isolement passager).

\subsubsection{Dégoût et utilisation abusive du texte de départ comme appât}

Dans le thriller Transgressions de Sarah Dunant, la traductrice Elizabeth Skvorecky est décrite dès le départ comme un personnage esseulé, anti-social, marginal, insomniaque et déprimé qui plonge peu à peu dans la folie suite aux intrusions d'un violeur dans sa maison. Le sexe sous toutes ses formes joue un rôle important dans ce roman qui paraît assez cru et d'un niveau peu élevé.

(13) Während ich dasass [sic], mich abquälte und seine Schlangensätze auf- und wieder zusammenrollte, damit nichts von dem beabsichtigten Glitzerspiel verloren ginge, sagte er obenhin und ganz nebenbei: ... ungenau, wie Frauen nun einmal sind. .. Ich erstarrte vor Wut. [...] Ungenau, wie Frauen nun einmal sind, schrieb ich also getreu und genau in meiner Muttersprache nieder, stand auf und rannte in die Küche. (AS pp. 130). Le texte en gras est seulement en italique dans l'original. Traduction A. Siouda.

(14) Nun, fuhr A.S. fort, weiterhin ohne mich anzublicken, bei Frauen erkennt man viel deutlicher, von welchem Tier sie herstammen, denn dieser Ursprung ist nicht wie beim Manne durch Willen, Bewusstsein und einen harten Lebenskampf verwischt worden.

Ich schleuderte die Ausgabe der Biblioteca Adelphi Band 70 gegen das Fenster. (AS pp. 132). Traduction A. Siouda. 
Le travail de la traduction permet à Elizabeth de s'isoler totalement, ce qui lui convient, mais en même temps, elle dénigre sa propre activité : "Ainsi, qu'est-ce qu'elle avait à la place? Quatre-vingt-mille mots de porno anglais sur un ordinateur et des taches de sperme d'un violeur-amant [...] sur sa robe "(15)) et elle traduit faute de mieux : "Sa traduction lui prendrait le reste de l'année et une partie de la nouvelle. Pourquoi pas ? Elle n'avait rien de mieux à faire de sa vie ".(16) En fait, elle ne se sent à l'aise qu'avec les mots des autres (le texte de l'auteur) et ne parvient pas à s'exprimer.

Elle-même, elle n'avait rien à dire. Ou rien qui, à ses yeux, aurait pu intéresser quelqu'un. Maintenant, en tant que traductrice, elle avait la confiance de jouer avec les mots des autres, mais elle était encore trop souvent maladroite quand il s'agissait de trouver les siens. (17)

Comme Möbius dans le texte de Wolter, Elizabeth entre quasiment en osmose avec le texte qu'elle traduit et son auteur. Comme A.S., l'auteur semble ici devenir un partenaire invisible qui la surveille : "Ceci lui procurait un plaisir tranquille, comme lorsque l'on travaille en phase avec un partenaire invisible, qui se tient là à votre épaule, vous surveille, vous chuchote à l'oreille „(18). En plongeant totalement dans le texte, Elizabeth finit par s'identifier à la victime (qui subit des atrocités sexuelles dans le texte), un peu comme Éléonore dans le texte de Rioux. Dans sa vie réelle, Elizabeth se fait effectivement violer chez elle. Lors de ce viol, elle ressent une certaine volupté, tandis que le violeur s'en va en pleurant. Elle semble sortir de cette expérience en "vainqueur " et c'est pourquoi elle appréhende le retour du violeur. Avec des bribes de brouillons de ses traductions dans lesquels elle change intentionnellement le texte de départ qu'elle utilise comme appât (en les plaçant dans sa poubelle pour que le violeur les lise), elle essaie de lui tendre un piège qui échoue. La police la protège pendant une nuit, mais le violeur vient à un autre moment et elle réussit à le tuer in extremis.

Évidemment, compte tenu de l'intrigue, l'association traducteur/auteure paraît impossible. On retrouve dans ce roman le cliché du sadisme mâle et de la victimisation de la femme, même si Dunant prétend montrer une certaine supériorité de la femme (son héroïne ne reste pas passive, ne se laisse pas faire, tend des pièges) et se défend, en tant que féministe, d'avoir écrit un texte misogyne. Reste à savoir comment le public perçoit ce texte et à ce titre, Horeck cite le journal The Observer qui écrit le 1er juin 1997 «Dunant, une féministe autoproclamée, avait

(15) So what did she have instead? Eighty thousand words of English sleaze on a computer and semen stains on her robe from a rapist lover [...] - (TG p. 246). Traduction A. Siouda.

(16) Its translation would take her the rest of the year and into the new one. Why not? She had nothing better to do with her life. (TG p. 9). Traduction A. Siouda.

(17) She herself had had nothing to say. Or nothing she thought anyone would be interested in. Now, as a translator she had the confidence to play with other people's words, but was still too often wrong-footed when it came to finding her own. (TG p. 14). Traduction A. Siouda.

(18) There was a quiet pleasure to be had in this, like working in unison with an invisible partner at your shoulder, watching over you, whispering in your ear ". (TG p. 38). Traduction A. Siouda. 
écrit un roman incluant un des scénarios pornographiques parmi les plus anciens qui puissent exister : une scène de viol lors de laquelle la femme finit par jouir de l'expérience "(19).

\subsubsection{Idéalisation de l'auteur et du texte de départ}

Dans le roman Juste à la porte du jardin d'Eden de Patricia Reznikov, le processus de traduction est certes mentionné, mais l'histoire tourne surtout autour d'un groupe d'amis trentenaires perturbés par l'arrivée d'une mystérieuse étrangère appelée Nadja. Colomba, la traductrice et narratrice qui fait partie de ce groupe est présentée comme une personne seule, manquant de confiance en elle et extrêmement angoissée. " II est vrai que je passe auprès de mes amis comme une spécialiste de l'angoisse " (JPE p. 21). Elle aussi dénigre son travail de traduction, d'autant plus qu'elle est également écrivaine, mais sans le moindre succès. Dans son groupe d'amis hétéros et, pour certains, homosexuels, elle ne fait pas partie des couples qui s'y sont formés. "Quelle effrayante solitude, me dis-je, en pensant à ma vie. " (JPE p. 122). Dans cette solitude, elle se met à aduler un auteur américain, Paul Ashburn et sa poésie, déjà traduite. Elle se met en tête de traduire l'unique roman de cet auteur avant même de l'avoir lu. Elle reçoit le livre de l'auteur lui-même, ainsi qu'une lettre, et la lecture de ce récit autobiographique intitulé Nora prend une place énorme dans la vie de Colomba.

L'objet-livre prend une importance démesurée : elle l'emporte partout, le met sous son oreiller et en fait un porte-bonheur. Plus que sur le processus de la traduction, sa réflexion porte sur la création et les parallèles qu'elle établit entre sa propre vie et celle d'Ashburn. Le récit imbriqué (le texte de l'auteur fictif Ashburn) que le lecteur découvre avec Colomba est tout sauf captivant, et l'attachement de Colomba pour ce texte paraît étrange. Colomba elle-même se sent comme une étrangère sur terre et son malaise psychique se matérialise en saignements abondants et médicalement inexpliqués par tous les orifices possibles, un fait qui l'isole encore plus socialement. La relation qu'elle entretient avec l'auteur reste largement imaginaire, car en réalité, leur correspondance (États-Unis - France) se résume à trois lettres échangées. II est à noter par contre que Colomba s'imagine non seulement une relation d'amour entre une traductrice et son texte à traduire, mais aussi entre l'éditeur et le texte d'origine : " II faudrait dénicher l'éditeur capable d'avoir une vraie relation d'amour avec Nora " (JPE p. 257). Finalement, le mandat de traduction pour le roman Nora lui échappe, car quelqu'un d'autre s'empare du projet. Quant à Ashburn, Colomba ne le rencontre pas puisqu'il meurt aux États-Unis avant qu'elle ne s'y rende.

Comme Reznikov établit parfois des parallèles entre création artistique et biologique, il est évident qu'elle a choisi une héroïne traductrice sans enfant qui cherche, en vain, à compenser ce manque par l'art. Ses propres projets d'écriture n'aboutissent pas et elle ne parvient pas à considérer ses traductions comme un acte créatif.

(19) Dunant, a self-identified feminist, had written a novel including one of the oldest pornographic scenarios in the book: a rape scene in which a woman ends up enjoying the experience. " (Horeck 2000 : 262). Traduction A. Siouda. 


\subsection{La traductrice comme métaphore et artiste de la relation}

\subsubsection{La traductrice-médiatrice dans la famille}

Le roman assez court de Barbara Frischmuth Die Ferienfamilie a été réédité en 2001, preuve qu'il ne manque pas d'actualité, même vingt ans après sa première parution. La traductrice Nora n'établit aucune relation entre texte d'origine et/ou son auteur, et le livre ne présente pas de réflexion sur le processus de traduction. Le métier de la traductrice sert uniquement d'élément de construction pour l'histoire, il est au service du récit. Une femme indépendante (divorcée) et active part en vacances dans les montagnes avec trois enfants pour y travailler pendant plusieurs semaines. Le travail concret est décrit de façon assez idyllique, et la traductrice travaille encore avec dictionnaire, cahiers et stylo. En plus, dans son cadre idyllique, elle avance à pas de géant dans sa traduction.

Le côté relationnel se retrouve concrètement dans les rapports de Nora avec son fils Pu, le fils d'un premier mariage de son ex-mari, Fenek, et sa nièce Laja. À notre époque où il y a énormément de familles recomposées, une telle association n'étonne plus.

Nora est une excellente communicatrice et elle joue en quelque sorte la médiatrice entre les enfants et leurs parents et leur offre, en outre, une certaine stabilité affective en les réunissant dans cette Ferienfamilie (famille de vacances).

Ses capacités de médiatrice vont aussi au-delà de la relation adultes/enfants. En effet, Nora a aussi un rôle de médiatrice entre hommes et femmes (qui ne partagent pas les mêmes valeurs) et entre les habitants de la ville et de la campagne (dont les visions conservatrices s'opposent à l'ouverture d'esprit de Nora) (cf. Resch 2005).

Malgré une certaine solitude due à son statut de femme divorcée, Nora joue un rôle très positif. Grâce à sa capacité de traduction métaphorique/de médiation, elle est une véritable artiste de la relation, bien dans sa peau, et qui n'a pas besoin d'établir une relation virtuelle et quasi pathologique avec un auteur réel ou imaginaire, car elle vit une relation réelle avec un ancien camarade de classe retrouvé pendant les vacances.

\subsubsection{La traductrice-médiatrice entre cultures musulmane et chrétienne}

La traductrice Sammar dans le roman The Translator de Leila Aboulela contraste fortement avec les protagonistes décrits ci-dessus. II s'agit en effet d'un personnage positif contribuant à la médiation spirituelle et culturelle et qui trouve non seulement son propre bonheur mais contribue aussi à celui des autres.

La mélancolie et la profonde tristesse qu'elle ressent au début du roman sont dues à son deuil.

Elle a perdu son mari dans un accident à Aberdeen et depuis, son fils est élevé à Khartoum, dans sa belle-famille, tandis qu'elle mène une vie recluse en Écosse. Seul son travail de traduction ainsi que sa foi (et la pratique rituelle) la maintiennent en vie. En ce sens, elle se 
différencie des autres traductrices analysées, car grâce à sa foi, elle ne semble jamais complètement perdue ou folle, même si, parfois elle se sent comme une somnambule dans sa vie et se laisse aller à la déprime et à la négligence.

Le processus de traduction n'est mentionné qu'en marge, car la vraie Über-Setzung, ce passage "d'une rive à une autre ", a lieu du point de vue religieux et culturel dans ce texte. C'est grâce à Sammar que le lecteur/la lectrice a accès à un point de vue intériorisé et très intime d'une musulmane convaincue et c'est certainement l'intention d'Aboulela : montrer et faire comprendre sa foi profonde en l'islam à un public occidental et, éventuellement, l'en convaincre. Tel est, en fait, l'espoir de la traductrice Sammar qui tombe amoureuse de l'islamologue britannique Rae : elle espère qu'il se convertisse pour qu'elle puisse se marier avec lui. Un espoir qui va finalement se réaliser, mais non sans mal.

Par ailleurs, Sammar est une médiatrice et Über-Setzerin pour les gens du monde occidental comme pour les gens en Afrique. Ainsi, elle s'efforce de faire comprendre à son propre frère Waleed à Khartoum que l'idée de la vie facile en Europe n'est qu'un cliché et que la réalité est tout autre.

Par rapport au processus de traduction, il est à noter que l'héroïne précise qu'elle veut traduire "like a pane of glass ", ce qui correspond à la conception traditionnelle en traductologie, alors qu'en tant que médiatrice entre les cultures et religions, la traductrice est très présente. Le côté didactique, religieux et engagé qui pourrait éventuellement gêner le public occidental est indéniable.

\section{Conclusion}

En raison du corpus restreint du mémoire, il est impossible de tirer des conclusions ayant une quelconque valeur statistique. Néanmoins, à la lumière du concept d'habitus repris par Kaindl (en référence à Bourdieu), il est possible de relever des dimensions physiques, psychiques et cognitives communes chez les sept traductrices protagonistes:

- Dimension physique : la traductrice est nue (Marine), souffre de saignements mystérieux (Colomba), est surmenée (Möbius), se sent malade et comme une somnambule (Sammar).

- Dimension psychique : la traductrice souffre d'un manque d'estime de soi, de culpabilité (Marine, Éléonore, Elizabeth, Colomba) ; elle est esseulée (Marine, Éléonore, Elizabeth, Colomba, Nora, Sammar) ; elle est angoissée (Éléonore, Elizabeth) ; elle est déprimée (Elizabeth, Colomba, Sammar) ; elle est dépressive et suicidaire (Éléonore) ; elle est folle (Elizabeth) et vue ainsi par son entourage.

- Dimension cognitive : le processus de traduction constitue le sujet principal (Marine, Éléonore, Möbius, Elizabeth) ; la traduction est vue surtout comme un gagne-pain (Colomba, Nora, Sammar), la traduction est utilisée métaphoriquement (Nora, Sammar) ; la fidélité 
absolue face à l'original sacré est relevée et respectée (Marine, Éléonore, Sammar) ou respectée et traitée avec ironie (Möbius) ; le texte de départ est utilisé à des fins personnelles (Elizabeth).

On l'a vu, les traductrices fictives sont bien souvent des êtres souffrants et psychologiquement fragiles, voire aliénés. À ce niveau, elles ont bien des choses en commun avec leurs homologues fictifs masculins.

En outre, je constate sur la base de mon analyse que, dans les ouvrages portant sur le processus de traduction, la traductrice et la traduction ont toujours un rôle subordonné à l'autorité de l'auteur et de l'original. La représentation de la traductrice est donc doublement négative, d'une part à cause de son activité inférieure et d'autre part à cause de son sexe (considéré comme inférieur).

Le texte de Wolter, particulièrement réussi, fait exception. Wolter dépeint une sorte d'alter ego à travers son personnage de traductrice. Möbius a de véritables relations sociales. Elle affronte un texte de départ à caractère misogyne avec une sensibilité féministe et une ironie libératrice. A.S. constitue ainsi une sorte de "métatexte dissimulé " et une production créative permettant à la traductrice réelle, Christine Wolter, de mener une réflexion critique sur le processus de traduction et d'exprimer des réactions dont elle a évidemment dû s'abstenir pendant son travail de traduction rémunéré.

Les Über-Setzerinnen Nora et Sammar qui ne traduisent pas de textes littéraires se démarquent nettement des autres héroïnes traductrices, malgré leur solitude, car elles évoluent en tant qu'artiste de la relation (Nora) ou médiatrice culturelle (Sammar) aidant les autres à trouver leur bonheur.

Ce qui frappe le plus, dans l'analyse de ces textes se rapportant à des traductrices littéraires, c'est que seules des femmes entrent en relation avec l'auteur du texte de départ ou avec ce texte lui-même. Une analyse plus exhaustive permettrait de déterminer si cette situation se retrouve dans d'autres textes ou s'il existe des personnages de traducteurs hommes entrant en relation avec une auteure fictive et/ou avec son texte. Par ailleurs, s'il devait ressortir d'une telle étude que, dans la fiction, seules les traductrices nouent de telles relations, cette capacité reflète-t-elle un besoin inné de la femme, un comportement genré et donc appris ou un talent exceptionnel ? Ou bien, faut-il conclure que les auteures réelles, qui ont une expérience en tant que traductrices, n'attribuent le rôle du traducteur qu'à des protagonistes féminins parce qu'elles ont totalement intériorisé l'image négative et misogyne de la traductologie traditionnelle et patriarcale ainsi que le cliché de la position secondaire de la traduction - et de la traductrice - au point qu'elles n'osent même pas s'y opposer dans leur imagination d'auteure ? Finalement, les romans présentant une association auteurE/traducteur seraient-ils encore à écrire? 


\section{Bibliographie}

\section{Corpus}

Aboulelea, Leila (1999) : The Translator, Black Cat. New York, 203 p.

Dunant, Sarah (1998) : Transgressions, Warner Books, London, 375 p.

Frischmuth, Barbara (2001) : Die Ferienfamilie, 1. Auflage. Aufbau Taschenbuch Verlag GmbH, Berlin, (Erste Auflage 1981), 102 p.

Poulin, Jacques (2006) : La traduction est une histoire d'amour, Leméac/Actes Sud, Montréal, $132 \mathrm{p}$.

Rioux, Hélène (1995) : Traductrice de sentiments, XYZ éditeur, Montréal, 173 p.

Reznikov, Patricia (2001) : Juste à la porte du jardin d'Eden, Mercure de France, Sans indication de lieu. 299 p.

Wolter, Christine (1988) : A.S., Dans : Piazza Brà. Erzählungen, Zürich, Benziger, 35 p.

\section{Ouvrages et articles de recherche}

ANDRES, Dörte (2008) : Dolmetscher als literarische Figuren. Von Identitätsverlust, Dilettantismus und Verrat, München.

BOREK, Johanna (1996) : " Der Übersetzer ist weiblich und damit unsichtbar. Übersetzen als Herrschaftsverhältnis, unter anderen ", Quo vadis Romania? 7, p. 27-33.

BRISSON, Josée (2008) : La traduction, ça se soigne, Circuit, la santé mentale des langagiers, $\mathrm{n}^{\circ} 101$, p. 14.

DELISLE, Jean (2002) : Portraits de traductrices, Presses de l'université d'Ottawa, Ottawa.

DELISLE, Jean (2003) : "Les traducteurs de papier : un portrait réaliste? ", Circuit, $\mathrm{n}^{\circ} 81$, p. 18-19.

HORECK, Tanya (2000) : " "More Intimate Than Violence”: Sexual Violation in Sarah Dunant's Transgressions ". Women: A Cultural Review, Volume 11, Issue 3, October, p. 262-272.

KAINDL, Klaus (2005) : " Einleitung ». Klaus Kaindl, Ingrid Kurz (éd.), Wortklauber, Sinnverdreher, Brückenbauer?, Wien, p. 9-18.

KAINDL, Klaus/KURZ, Ingrid (2008a) : "Einleitung ". Dans : Helfer, Verräter, Gaukler, Das Rollenbild von Translatorlnnen im Spiegel der Literatur, Wien, p. 9-18. 
KAINDL, Klaus (2008b) : «Zwischen Fiktion und Wirklichkeit: Translatorlnnen im Spannungsfeld von Wissenschaft, Literatur und sozialer Realität ". Larisa Schippel (Hg.), Translationskultur ein innovatives und produktives Konzept, Berlin, p. 307-333.

MILANO APPEL, Anne (2003) : "Behind the Mirror: Now We See Her, Now We Don't. " The ATA Chronicle, avril, p. 28-33.

NUTTING, Stéphanie (2005) : "Hélène Rioux : traductrice géographe ". Agnes Whitfield (éd.) Le métier du double : portraits de traductrices et traducteurs littéraires, Québec, p. 246-263.

RESCH, Renate (2005) : "Das Patchwork übersetzen. Barbara Frischmuths Die Ferienfamilie ". Klaus Kaindl, Ingrid Kurz (éd.), Wortklauber, Sinnverdreher, Brückenbauer?, Wien, p. 213-221.

SAINT-MARTIN, Lori (2007) : Romans d'homme, voix de femme "Marie Auger ", Gilles Archambault, Jacques Poulin et Maxime Mongeon. Voix et Images, volume 32, $n^{\circ} 2$ (95), hiver, p. 31-47.

\section{Articles sur internet}

http://webcache.googleusercontent.com/search?q=cache:v7SOYbBWXall:www.usherbrooke.ca/ grelq/recherche/projets-de-recherche/les-traducteurs-fictifs-dans-la-litterature-quebecoise-

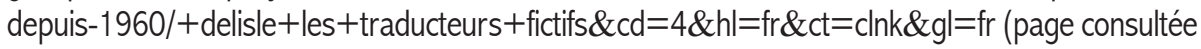
le 16/03/2012)

XYZ éditeur http://www.xyzedit.qc.ca/communique.php?lang=fr\&id=169 page consultée le 23/08/2009

Anja Siouda est traductrice, diplômée de l'École de traduction et d'interprétation de Genève (ETI), licenciée ès lettres et écrivaine. La version originale intégrale en langue allemande de son mémoire peut être consultée et téléchargée gratuitement sur le site des archives ouvertes de l'université de Genève : http://archive-ouverte.unige.ch/vital/access/manager/Repository/unige:12743?query=siouda Site internet de l'écrivaine : www.anjasiouda.com 